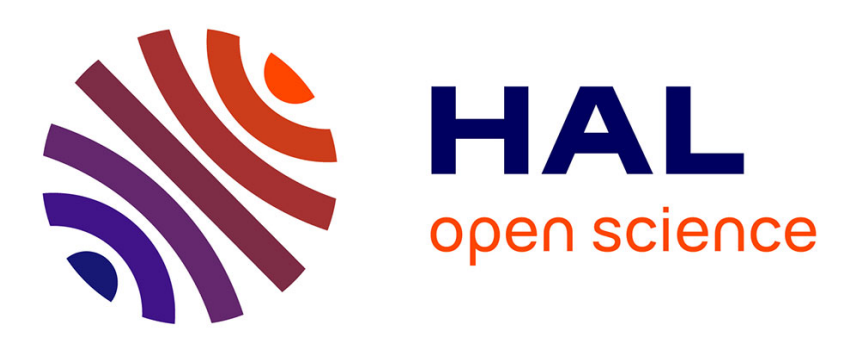

\title{
Assessment of evaporation equilibrium and stability concerning an acoustically excited drop in combustion products
}

Yves Mauriot, Roger Prud'Homme

\section{- To cite this version:}

Yves Mauriot, Roger Prud'Homme. Assessment of evaporation equilibrium and stability concerning an acoustically excited drop in combustion products. Comptes Rendus Mécanique, 2014, 342 (4), pp.240 - 253. 10.1016/j.crme.2014.02.004 . hal-01445115

\section{HAL Id: hal-01445115 https://hal.sorbonne-universite.fr/hal-01445115}

Submitted on 24 Jan 2017

HAL is a multi-disciplinary open access archive for the deposit and dissemination of scientific research documents, whether they are published or not. The documents may come from teaching and research institutions in France or abroad, or from public or private research centers.
L'archive ouverte pluridisciplinaire HAL, est destinée au dépôt et à la diffusion de documents scientifiques de niveau recherche, publiés ou non, émanant des établissements d'enseignement et de recherche français ou étrangers, des laboratoires publics ou privés. 


\section{Assessment of evaporation equilibrium and stability concerning an acoustically excited drop in combustion products.}

\section{By Yves Mauriot ${ }^{1}$ and Roger Prud'homme ${ }^{2}$}

\footnotetext{
${ }^{1}$ Research Engineer, ONERA - The French Aerospace Lab, F-92322 Châtillon, France, tel. + 331467343 34, yves.mauriot@onera.fr

${ }^{2}$ Emeritus Research Director, Institut Dalembert, UPMC/CNRS UMR 7190, case 162, 4 place Jussieu, 75252

Paris cedex 05, France, tel. +33 1442773 06, roger.prud_homme@upmc.fr
}

\section{Summary}

The evaporation of drops in a sound field has been the subject of numerous studies aimed at determining its role in combustion instability. The models generally assume local equilibrium evaporation at the interface. We determine here the conditions of validity of this assumption, without calling into question other a priori assumptions of the classical model, in particular spherically symmetric quasi-steady evolution in the gas phase and liquid phase thermal unsteadiness with pure heat conduction.

Another possible phenomenon concerns the differential recoil of the vapor. In the case of rapid evaporation a pressure difference appears between both sides of the interface, even if the latter is plane. This pressure difference, usually neglected, is proportional to the square of speed and the resulting force is oriented toward the denser fluid, i.e. the liquid. A very fast evaporation may even cause local deformation, i.e. Hickman instability. The stability condition concerning this phenomenon has also been determined.

This study was co-funded by CNES (French Space Agency) and ONERA and was performed in the framework of CNES-ONERA French Research \&Technology activities on the high frequency combustion stability of liquid propellant rocket engines.

Key-words: acoustic excitation; drop; evaporation; instability; non-equilibrium; vapor recoil

Mots-clés : déséquilibre ; évaporation ; excitation acoustique ; goutte ; instabilité ; recul de la vapeur 


\section{List of symbols}

$a, b, c \quad$ constant coefficients

$A, B \quad$ thermodynamic coefficients in the transfer function

$B_{M}, B_{T} \quad$ Spalding parameters for mass and temperature

$c_{p} \quad$ specific heat at constant pressure

C combustion chamber level

d droplet diameter

Da Damköhler parameter

$E(u, \theta)$ function $E(u, \theta)=1-(1+i) \sqrt{3 u / 2 \theta} \operatorname{coth}[(1+i) \sqrt{3 u / 2 \theta}]$

$g$ thermodynamic potential per unit mass, gravitational acceleration

$G \quad$ gas phase

$h \quad$ liquid height

$H \quad$ container height

$\mathrm{Hi} \quad$ Hickman number

$k \quad$ heat conductivity

$l \quad$ latent heat per unit mass

$L \quad$ liquid phase; phenomenological coefficient for near-equilibrium evaporation-condensation

$M \quad$ mass

$\mathcal{M} \quad$ molar mass

$\dot{m} \quad$ unit mass flow rate

$\dot{M} \quad$ mass flow rate for an evaporating droplet

$N \quad$ response factor $N=\bar{\alpha} \mathfrak{R}(Z)$

$\overrightarrow{\mathbf{N}} \quad$ unit normal at a point of an interface; quantity $\vec{\nabla} \cdot \overrightarrow{\mathbf{N}}$ being the average normal curvature

$p \quad$ thermodynamic pressure

$p_{\text {sat }} \quad$ saturation pressure

$q_{T} \quad$ coefficient equal to $\tau_{T} / 6 \pi \tilde{\tau}_{T}$

$r \quad$ gas constant per unit mass $r=R / \mathcal{M}$

$\bar{r}_{s} \quad$ mean radius of the fed droplet

$R \quad$ universal gas constant

$S \quad$ interface level; cross section area

$T \quad$ temperature in $\mathrm{K}$

$u \quad$ reduced pulsation: $u=3 \omega \bar{\tau}_{v}$

$V \quad$ speed

$\overrightarrow{\mathbf{v}} \quad$ fluid velocity

$\overrightarrow{\mathbf{w}} \quad$ interfacial velocity

We Weber number: $W e=\rho\left(V_{G}-V_{L}\right)^{2} / \sigma$

$x, y, z \quad$ Cartesian coordinates

$Z \quad$ transfer function of the fed oscillating droplet

$\alpha, \alpha_{v}, \alpha_{c}$ evaporation-condensation, vaporization, condensation coefficients respectively

$\bar{\alpha} \quad$ coefficient of the transfer function of the fed oscillating droplet

$-\beta_{L} \quad$ thermal gradient in a liquid boundary layer

$\delta \quad$ thickness of a boundary layer ( $\delta$ for the vapor side, $\delta_{L}$ for the liquid one)

$\lambda \quad$ heat conductivity

$\eta(x, y, t)$ function describing a disturbed surface $z=\eta(x, y, t)$, the reference value being zero

$\kappa \quad$ heat diffusivity

$\mu \quad$ dynamic viscosity; thermodynamic potential per mole $\mu=\mathcal{M} g$

$\mu_{j} \quad$ chemical potential of species $j$

$v \quad$ quantity equal to: $(1+i) \sqrt{3 u / 2 \theta}$ 


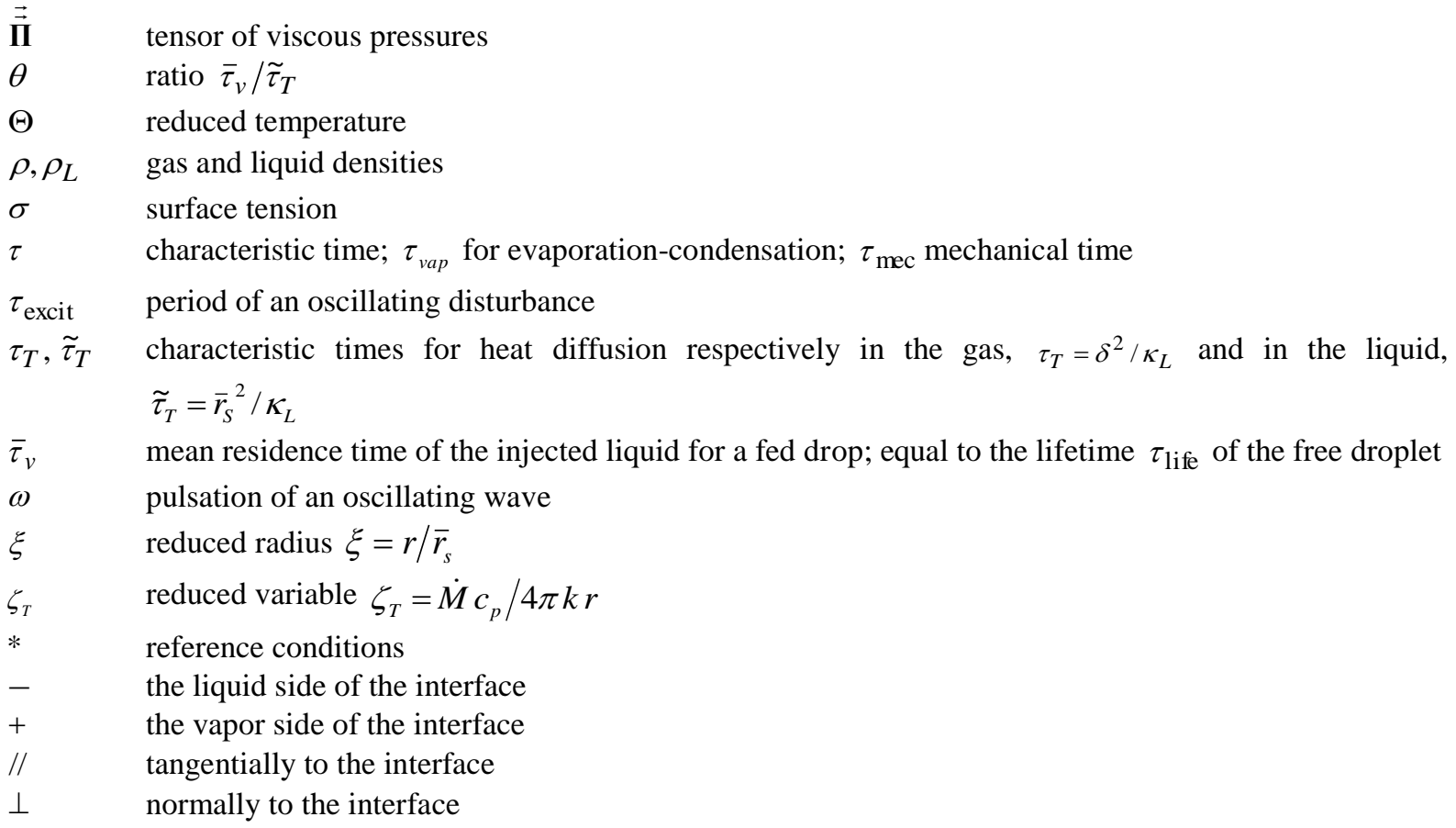

\section{Introduction}

The evaporation of drops in an acoustic field has been the subject of many studies aimed at determining its role in combustion instability. The models generally assume local evaporation equilibrium at the interface. We want to determine here the conditions of validity of this assumption, without questioning the other a priori assumptions of the classical model, in particular: spherical symmetry, quasi-stationary evolution in the gas phase and thermal unsteadiness of the liquid phase with pure heat conduction [1, 2].

The local evaporation equilibrium is characterized by the equality of chemical potentials of the constituent of the drop in liquid and vapor phases,

$$
\mu_{F L}=\mu_{F V}
$$

In the case of local evaporation non-equilibrium, the equality of chemical potentials is no more guaranteed and the mass flow rate of vaporization is a function of their difference. According to Bond and Struchtrup [3], in the case of a pure substance, the rate of evaporation can be written

$$
\dot{m}=\left(\frac{\mathcal{M}}{2 \pi R}\right)^{1 / 2}\left(\alpha_{v} \frac{p_{s a t}}{\left(T_{S}\right)^{1 / 2}}-\alpha_{c} \frac{p^{+}}{\left(T^{+}\right)^{1 / 2}}\right),
$$

with two different coefficients $\alpha_{v}, \alpha_{c}$, respectively for evaporation and condensation, $\mathcal{M}$ the molar mass, $\mathrm{R}$ the universal gas constant, $p_{\text {sat }}$ the saturation vapor pressure at the temperature $T^{-}=T_{S}$ of the liquid surface, $T^{+}, p^{+}$corresponding to the gas. Coefficient values $\alpha_{v}, \alpha_{c}$ become equal when one tends towards equilibrium: $\alpha_{v}=\alpha_{c}=\alpha$.

Another phenomenon can occur during very fast evaporation, changing the shape of the liquid-vapor interface. It is called the vapor recoil (see Appendix A.1). It can be explained by the momentum balance at the interface and results in a force acting towards the densest fluid, i.e. the liquid. 
The case of vacuum evaporation, which results in surface deformation, has been particularly studied by Palmer [4], who found the stability limit of the phenomenon. This is the Hickman instability [5] (see Appendix A.2).

We study here the evaporation of drops in a combustion chamber that is disturbed by high frequency acoustic fluctuations (at frequencies from $250 \mathrm{~Hz}$ to $20000 \mathrm{~Hz}$ ). Indeed, it is important to characterize the regime of evaporation in order to properly model the evaporation and combustion instability phenomena.

Our goal is to predict the existence of an evaporation out of equilibrium. We do not intend to introduce immediately this disequilibrium into the equations to solve them. Indeed, the local evaporation disequilibrium may considerably complicate the numerical resolution. It is however important to find a criterion of occurence. For this purpose, we will compare the characteristic times and length scales.

The relaxation of evaporation from a non-equilibrium state depends on the mechanical and thermodynamic evolution of the fluids. One has to compare a characteristic time of this evolution to the characteristic time of evaporation; the resulting comparison will lead to a near-equilibrium criterion through a Damköhler number. So the local evaporation non-equilibrium can be characterized, in quasi-stationary mode, by the Damköhler parameter of local evaporation defined by the ratio of a mechanical time $\tau_{\text {mec }}$ to a vaporization time $\tau_{\text {vap }}$.

The instability of recoil involves also the previous characteristic times, but the resulting expression of the Hickman number also depends on a reference flow rate and on its temporal derivative. We will study this instability assuming local evaporation equilibrium and will assume that the Palmer's results remain valid with this hypothesis and also for spherical drops in the reference state.

In order to determine evaporation quantities of a flat layer, we will use on the one hand a specific simple model and on the other hand quantities related to the evaporation of a drop, and put to good use our knowledge of the Heidmann model concerning a droplet fed by a steady flow [1] (see Appendix A.3).

\section{Condition of evaporation equilibrium}

\subsection{Characteristic time of the evaporation process}

So as to determine first the characteristic time of the evaporation process at constant volume, we imagine a cylindrical container of height $H$. The liquid height is $h$ and the cross section area $S$ (Figure 1).

In the case of a single coefficient $\alpha$ for evaporation and condensation, the flow rate per unit area is

$$
\dot{m}=\alpha\left(\frac{\mathcal{M}}{2 \pi R}\right)^{1 / 2}\left(\frac{p_{s a t}}{\left(T_{s}\right)^{1 / 2}}-\frac{p^{+}}{\left(T^{+}\right)^{1 / 2}}\right) .
$$

Writing $p^{+}=p, T^{+}=T$ and assuming a uniform temperature,

$\dot{m}=\frac{\alpha}{\sqrt{2 \pi r T}}\left(p_{\text {sat }}-p\right)$ with $r=R / \mathcal{M}$. If $p<p_{\text {sat }}$, evaporation occurs.

Another relationship characterizes the total mass: $M=M_{G}+M_{L}=\rho_{L} h S+\rho_{G}(H-h) S=C t e$.

Sufficiently far below the critical point $\rho_{G} \ll<\rho_{L}$, so $M \approx S\left(\rho_{L} h+\rho_{G} H\right)$. On the other hand, assuming an ideal gas: $\rho_{G}=p / r T$.

At evaporation-condensation equilibrium, we have: $p=p_{\text {sat }}(T), h=h_{e}$, and: $M \approx S\left(\rho_{L} h_{e}+p_{\text {sat }} H / r T\right)$, from what we deduce $h \cong h_{e}-\frac{p-p_{\text {sat }}}{\rho_{L} r T} H$.

To study the near equilibrium evolution, $\rho_{L}=C s t$ is assumed. Thus we can write $S \dot{m}=-\frac{d M_{L}}{d t}=-\rho_{L} S \frac{d h}{d t}$. Given the conservation of the total mass we obtain the evolution equation of the

\footnotetext{
${ }^{1}$ We assume the ratio $S / H^{2}$ to be sufficiently small so as to make negligible any internal fluid motions as vortices, and thus to keep the one-dimensional behavior of the system.
} 
liquid level, $\frac{d h}{d t}+\frac{\alpha}{H} \sqrt{\frac{r T}{2 \pi}}\left(h-h_{e}\right)=0$, which provides $h=h_{e}+\left(h_{0}-h_{e}\right) e^{-t / \tau_{v a p}}$, with a relaxation time of phase change (evaporation or condensation)

$$
\tau_{\mathrm{vap}}=H \sqrt{2 \pi} /(\alpha \sqrt{r T})
$$

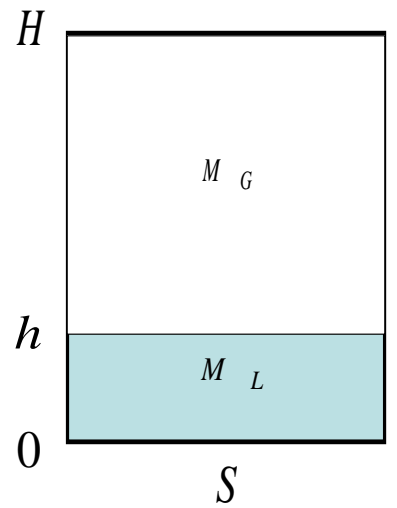

Figure 1. Closed and constant volume isothermal evaporation vessel.

To represent the case of drop evaporation, one has to replace $H$ by a characteristic length. Assuming $T=C t e$, the equation of the variable $h$ is verified also by the variable $p-p_{\text {sat }}$, so that: $p=p_{\text {sat }}+\left(p_{0}-p_{\text {sat }}\right) e^{-t / \tau_{\text {vap }}}$.

\subsection{Diffusion characteristic time}

Some "mechanical time" has to be compared to the previous evaporation time, in order to characterize the evolution of the system from an evaporation disequilibrium. The time of thermal diffusion plays this role; we have $\tau_{\text {méc }}=\tau_{T}=\delta^{2} / \kappa$ (where $\delta$ is a diffusion thickness and $\kappa$ is the thermal diffusivity).

For the flat layer at rest, the thickness of thermal diffusion in the gas is: $\delta=H$, if one assumes that the heat input comes from the top of the container.

In the case of an evaporating droplet (Appendix A.3) of diameter $\bar{d}$, supplied by a steady flow, we will estimate the thickness of thermal diffusion in the gas, using the temperature gradient at the interface. Thermal gradients that are external to the drop are considered as being reduced to those of the steady state ${ }^{2}$. In quasistationary regime with constant physical properties, and assuming spherical symmetry, the temperature $\bar{T}$ of the gas phase is a function of the radius $r$ only. With $\xi=r / \bar{r}_{S}$, we obtain

$$
\bar{T}=\frac{\bar{T}_{S}\left(1+\bar{B}_{T}\right)-\bar{T}_{\infty}}{\bar{B}_{T}}+\frac{1+\bar{B}_{T}}{\bar{B}_{T}}\left(\bar{T}_{\infty}-\bar{T}_{S}\right)\left(1+\bar{B}_{T}\right)^{-\frac{1}{\xi}}
$$

Details of the proof: The QS thermal profile in the gas phase is of the form (see Appendix A.3 and ref. [2]): $T=a_{T}+b_{T} e^{-\zeta_{T}}, \zeta_{T}=\overline{\dot{M}} c_{p} / 4 \pi k r$, with $\overline{\dot{M}}=4 \pi \frac{k}{c_{p}} \bar{r}_{S} \ln \left(1+\bar{B}_{T}\right)$, so $\zeta_{T}=\frac{1}{\xi} \ln \left(1+\bar{B}_{T}\right)$,

\footnotetext{
${ }^{2}$ Small high frequency perturbations change only a little these gradients; changes become important only in unstable frequency domain corresponding to $0 \leq u \leq u_{c}$ (see Annex A.3), and if disturbances are not small. In this case we go into the nonlinear domain, which is not studied here.
} 
i.e., $T=a_{T}+b_{T}\left(1+\bar{B}_{T}\right)^{-\frac{1}{\xi}}$. The boundary conditions $\bar{T}=\bar{T}_{S}$ at the surface of the drop and $\bar{T}=\bar{T}_{\infty}$ at infinity provide values of the constants $a_{T}, b_{T}$, hence the expression of $\mathrm{T}$ above.

We have:

$-\bar{\Theta}=\frac{\bar{T}-\bar{T}_{\infty}}{\bar{T}_{\infty}-\bar{T}_{S}}=\frac{\left(1+\bar{B}_{T}\right)}{\bar{B}_{T}}\left[\left(1+\bar{B}_{T}\right)^{-1 / \xi}-1\right]$, then: $\frac{d(-\bar{\Theta})}{d \xi}=\frac{\left(1+\bar{B}_{T}\right)^{\frac{\xi-1}{\xi}} \ln \left(1+\bar{B}_{T}\right)}{\bar{B}_{T} \xi^{2}}$.

At the droplet surface, $r=\bar{r}_{S}, \xi=1$, so $\frac{d(-\bar{\Theta})}{d \xi}=\frac{\ln \left(1+\bar{B}_{T}\right)}{\bar{B}_{T}}$.

The diffusion thickness in the gas thus writes :

$$
\left(\frac{\delta}{\bar{r}_{S}}\right)=\frac{\bar{B}_{T}}{\ln \left(1+B_{T}\right)}
$$

Figure 2 shows the evolution of the reduced temperature $\bar{\Theta}$ as a function of $\xi=r / \bar{r}_{S}$ for different values of the Spalding parameter ${ }^{3} \bar{B}_{T}=c_{p}\left(\bar{T}_{\infty}-\bar{T}_{S}\right) / \bar{l}$. The same study could be made about the concentrations. One observes that the temperature changes considerably in a region at the periphery of the droplet, which thickness has been characterized.

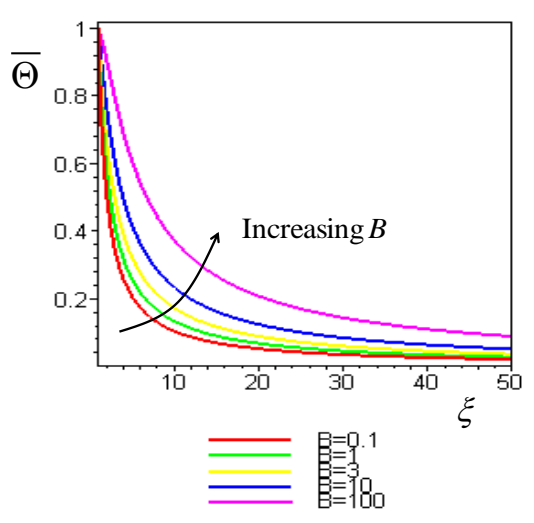

Figure 2. Evolution of the reduced temperature $\bar{\Theta}=\left(\bar{T}_{\infty}-\bar{T}\right) /\left(\bar{T}_{\infty}-\bar{T}_{S}\right)$ as a function of the reduced radius $\xi=r / \bar{r}_{S}$ for increasing values of the Spalding parameter $B=\bar{B}_{T}$ from 0.1 to 100 .

\subsection{Damköhler number of evaporation and condition of equilibrium}

To build a global criterion for evaporation equilibrium, we define first the Damköhler number of evaporation as the ratio or the diffusion time in the gas to the characteristic evaporation time, $D a=\tau_{T} / \tau_{\text {vap }}=(\alpha \delta / \kappa) \sqrt{r T / 2 \pi}$ and the corresponding condition of equilibrium is $D a>>1$. The quantities to be known are: the coefficient of evaporation $\alpha$, the thermal diffusivity of the gas $\kappa$, the gas temperature $T$, the diffusion thickness $\delta$ in the gas.

However, this condition ignores the disequilibrium caused by possible disturbances from the engine, assuming that the equilibrium be satisfied in the non-disturbed situation. So as not to affect this equilibrium, the

\footnotetext{
${ }^{3}$ Calculated at stabilized regime.
} 
period of disturbance $\tau_{\text {excit }}=2 \pi / \omega$ has to be much larger than the time of evaporation $\left(\tau_{\text {excit }}>>\tau_{\text {vap }}\right)$. We will write this equilibrium condition $\omega \tau_{T} / 2 \pi<<D a$.

Moreover, we have to consider a third condition, taking into account the characteristic lifetime of the drop, which is equal to the residence time $\bar{\tau}_{v}$ of the liquid injected into an equivalent fed drop. Indeed, reaching the equilibrium of evaporation during the major part of droplet lifetime implies that the vaporizing time be much shorter than the lifetime $\left(\bar{\tau}_{v} / \tau_{\text {vap }}>>1\right)$. This equilibrium condition may be written $\tau_{T} / \bar{\tau}_{v}<<D a$.

All conditions of evaporation equilibrium will thus be ensured by the following global criterion ${ }^{4}$, linking the four dimensionless ratios $D a, u, \theta$ and $q_{T}=\frac{1}{6 \pi} \frac{\tau_{T}}{\tilde{\tau}_{T}}$ :

$$
\operatorname{Da}>\sup \left(1, q_{T} \frac{u}{\theta}, q_{T} \frac{6 \pi}{\theta}\right)
$$

\section{Condition of Hickman instability}

Hickman instability can occur for high evaporation flow rates, e.g. for a plane layer of liquid under vacuum (see Palmer, 1976 [4], see also [6]). It is caused by local fluctuations of the recoil force. We introduce the Hickman number

$$
H i=\left(\frac{d \dot{m}}{d T}\right)^{*} \frac{\dot{m}^{*} \beta_{L} \delta_{L}^{2} \mu}{\rho_{L} \kappa_{L} \sigma^{*}}\left(\frac{1}{\rho}-\frac{1}{\rho_{L}}\right)
$$

which represents "the ratio of the destabilizing forces of differential vapour recoil and vapour viscosity to the stabilizing action of surface tension and thermal diffusivity" [4], with the following variables: $\delta_{L}$ the thickness of the liquid thermal boundary layer, $\left(-\beta_{L}\right)$ the thermal gradient in the same layer, $\dot{m}^{*}$ the evaporation unit flow rate in the reference situation, $\mu$ the dynamic viscosity of the gas, $\kappa_{L}$ the thermal diffusivity of the liquid, $\sigma^{*}$ the surface tension reference, $T$ the temperature, $\rho$ the density of the gas (the evaporated liquid), $\rho_{L}$ the liquid density. To estimate the Hickman number, we will assume a constant thermal time $\tau$ defined by $\frac{1}{\tau}=\frac{d(\ln T)}{d t}$, and consider the situation ${ }^{5}$ of Figure 1 . We have $\dot{m}^{*}=\frac{H p_{\text {sat }}}{r T \tau}\left(\frac{l}{r T}-1\right)$, because

$M_{L}=M-\frac{p_{\text {sat }}}{r T} H, \quad \dot{m}^{*}=-d M_{L} / d t=(H / r) d\left(p_{\text {sat }} / T\right) / d t \quad$ and for an ideal gas, assuming $\rho_{L} \gg \rho_{G}$, the Clapeyron relation writes $\frac{d p_{\text {sat }}}{d T} \cong \frac{l p_{\text {sat }}}{r T^{2}}$.

${ }^{4}$ Using the notations $\tilde{\tau}_{T}=\bar{r}_{S}^{2} / \kappa_{L}, u=3 \omega \bar{\tau}_{v}, \theta=\bar{\tau}_{v} / \tilde{\tau}_{T}$ (See Annex A3) and $q_{T}=\frac{1}{6 \pi} \frac{\tau_{T}}{\tilde{\tau}_{T}}$, we have on the one hand: $q_{T} \frac{u}{\theta}=\tau_{T} \frac{\omega}{2 \pi}=\frac{\tau_{T}}{\tau_{\text {excit }}}$. So $D a \gg q_{T} \frac{u}{\theta}$ means $\tau_{\text {excit }} \gg \tau_{\text {vap }}$. On the other hand, $q_{T} \frac{6 \pi}{\theta}=D a \frac{\tau_{\text {vap }}}{\bar{\tau}_{v}}$, so that $D a \gg q_{T} \frac{6 \pi}{\theta}$ means $\bar{\tau}_{v} \gg \tau_{\text {vap }}$.

\footnotetext{
${ }^{5}$ As the cause of the instability of Hickman depends on the situation (here the situation described by Figure 3), it will be necessary to re-consider the expression of the time $\tau$ to treat the case of a drop.
} 


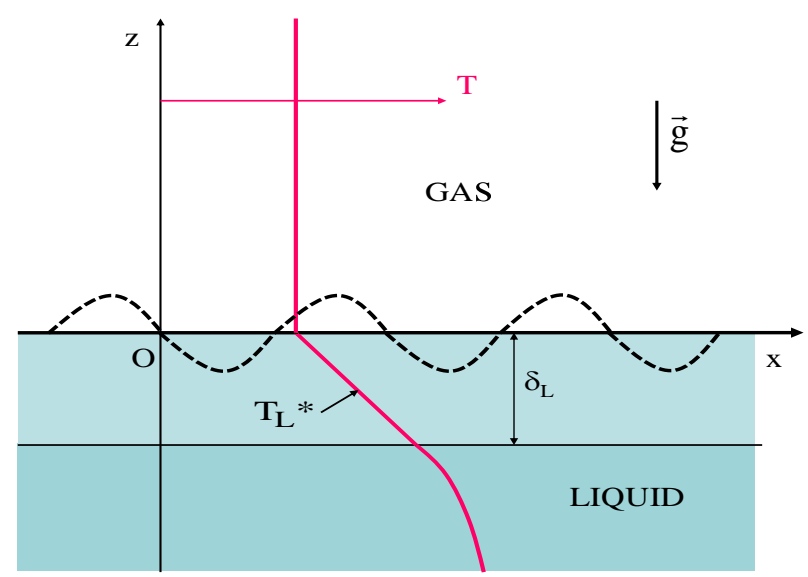

Figure 3. Configuration studied by Palmer.

Using the expression of the latent heat $l=\frac{b r T^{2}}{(T-c)^{2}}[1]$, we find $\left(\frac{d \dot{m}}{d T}\right)^{*}=\frac{H}{r \tau} \frac{p_{s a t}}{T^{2}}\left[\left(\frac{l}{r T}-1\right)^{2}-\frac{b c T}{(T-c)^{2}}\right]$.

Remarks: One may think to compare the former expression of $\dot{m}^{*}$ with the expression of $\dot{m}$ presented in annex A1.2, equation (21). The latter results from the evidence of fluxes and generalized forces in the expression of the entropy production rate and from a linearization, in the framework of classical thermodynamics of irreversible processes applied to a pure species. Studying evaporation in vacuum condition, Palmer (see annex A.2, equation (24)) uses a different but analogous expression of $\dot{m}$, with a generalized force $\left(\frac{p^{0}}{\left(T_{S}\right)^{1 / 2}}-\frac{p_{G}{ }^{*}}{\left(T_{G}{ }^{*}\right)^{1 / 2}}\right)$.

To evaluate the evaporation rate $\dot{m}^{*}$, we proceed here in a different way. Indeed, the assumed local evaporation equilibrium at the interface concerns the species constituting the liquid drop, which vapor is mixed with combustion products, as explained in annex A.3.

However, we take into account the recoil force, as Palmer does. This force results in our case from the high evaporation velocities encountered at high temperatures in rocket engine combustion chambers. The recoil force intervenes here in the expression of the Hickmann number given by Palmer, through the product $\dot{m}^{*}\left(\frac{d \dot{m}}{d T}\right)^{*}$ which depends from this force.

Substituting in the expression of the Hickman number, we obtain

$$
H i=\left(\frac{H p_{s a t}}{r T \tau}\right)^{2}\left(\frac{l}{r T}-1\right)\left[\frac{1}{T}\left(\frac{l}{r T}-1\right)^{2}-\frac{b(T+c)}{(T-c)^{3}}\right] \frac{\beta_{L} \delta_{L}^{2} \mu}{\rho_{L} \kappa_{L} \sigma^{*}}\left(\frac{1}{\rho_{V}}-\frac{1}{\rho_{L}}\right) .
$$

We will remove the index $(V)$ denoting the vapor and take $H=\delta$, the thickness of thermal diffusion layer in the gas. Considering that the acoustic disturbance is the cause of the possible Hickman instability, we evaluate the characteristic time $\tau$ of the thermal fluctuation at the droplet surface defined by $\frac{1}{\tau}=\left|\frac{d\left(\ln T_{s}\right)}{d t}\right|$, using $T_{s}^{\prime}=\frac{T_{s}-\bar{T}_{s}}{\bar{T}_{s}}$. Hence, setting $T_{s}^{\prime}=\hat{T}_{s} e^{i \omega t}$ and assuming $\left|\widehat{T}_{s}^{\prime}\right|<<1$ (which is verified in our application case), 
we get $\frac{1}{\tau}=\omega\left|\frac{\widehat{T}_{s}^{\prime} e^{i \omega t}}{1+\widehat{T}_{s}^{\prime} e^{i \omega t}}\right| \cong \omega\left|\widehat{T}_{s}^{\prime}\right|$. Moreover, as can be shown from the results presented in Annex A3, $\hat{T}_{s}=\frac{\hat{p}_{C}}{\bar{b}} \frac{A+B}{B-\theta E(u, \theta)}$. Then $\frac{1}{\tau} \cong \omega\left|\hat{p}_{C}\right|\left|\frac{1}{\bar{b}} \frac{A+B}{B-\theta E(u, \theta)}\right|$.

The thickness of the liquid thermal boundary layer $\delta_{L}$ and the temperature gradient $\beta_{L}=\left|d T_{l} / d r\right|$ in the liquid are calculated as follows in the case of a propellant droplet ${ }^{6}$.

Thermal gradients in the liquid are caused by high frequency disturbances from the combustion chamber that are transmitted by the gas to the whole drop or part of it. Indeed, the reference configuration is that of the steady state in which the temperature of the drop is uniform, unlike the gas temperature. Ideally, the thermal field is spherically symmetrical (Fig. 4) and produces density variations which cause convective motions.

Hypothesizing that we legitimately neglect the thermal convection due to the feeding of the droplet, we show hereafter that $\delta_{L} / \bar{r}_{S} \approx|1 / E(u, \theta)|$, with $E(u, \theta)=1-(1+i) \sqrt{\frac{3 u}{2 \theta}} \operatorname{coth}\left[(1+i) \sqrt{\frac{3 u}{2 \theta}}\right]$.

Indeed, setting $T^{\prime}{ }_{l}=\hat{T}_{l}(r) e^{i \omega t}$, we can demonstrate from the results presented in Annex A3 that $\hat{T}_{\text {lred }}=\frac{\bar{b}}{\hat{p}_{C}} \hat{T}_{l}=\frac{A+B}{B-\theta E(u, \theta)} \frac{\operatorname{sh}(v \xi)}{\xi s h v}$, where $v=(1+i) \sqrt{3 u / 2 \theta}, \xi=r / \bar{r}_{S}$. The modulus of the temperature
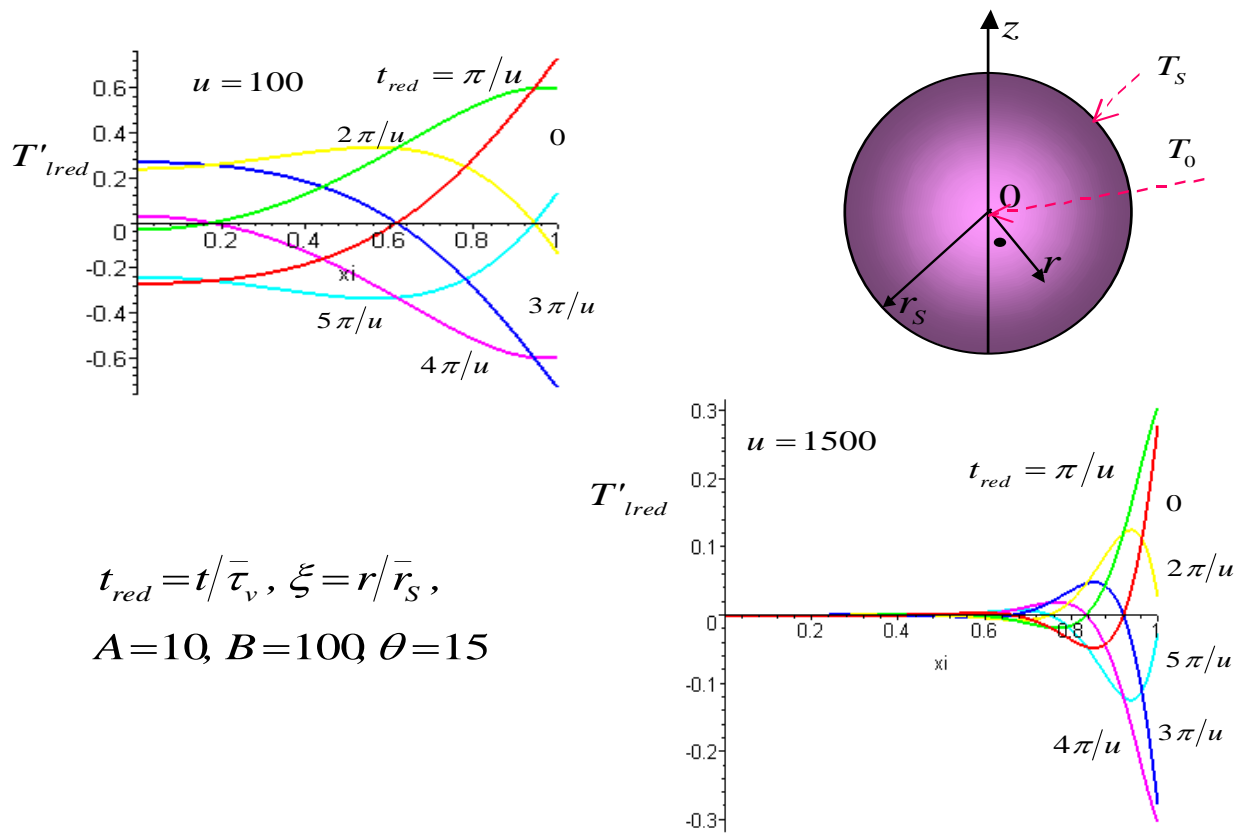

Figure 4. Two examples of the oscillating reduced temperature field inside the drop, resulting from a pressure perturbation in the chamber. They are depending of $\xi=r / \bar{r}_{S}$ for different values of dimensionless time $t_{r e d}=t / \bar{\tau}_{v}$. Increasing the excitation frequency leads to reduction of the wave penetration depth.

\footnotetext{
${ }^{6}$ Remark : Note that in his calculation, Palmer studied the Hickmann instability starting from an evaporation configuration out of local equilibrium, which is not our case here. On the other hand, the fact that he treated a vacuum evaporation led him to consider only the boundary layer in the liquid (Figure 3 ). We chose to do like this author.
} 
perturbation $\left|T^{\prime}{ }_{l}\right|$ is thus proportional to the quantity $\Theta_{l}=\operatorname{sh}(v \xi) / \xi \operatorname{sh} v$. A limited expansion of $\Theta_{l}$ near the surface gives $\Theta_{l}=1+(v \operatorname{coth} v-1) \Delta \xi$.

We find therefore $\Theta=0$ for $|\Delta \xi|=\delta_{L} / \bar{r}_{S}=|1 /(1-v \operatorname{coth} v)|=|1 / E(u, \theta)|$. In Fig. 5 are shown the results obtained with different values of $\theta$.

Let us notice that $|v \operatorname{coth} v|$ must be large enough, so that $|\Delta \xi|$ be less than 1. In this case we will have $|1 /(1-v \operatorname{coth} v)| \approx|t h v / v| \approx|1 / v|$ and thus $|\Delta \xi| \approx|1 / v| \propto \sqrt{\theta / u}$, i.e., $\delta_{L} / r_{S} \propto \sqrt{\theta / u}$.

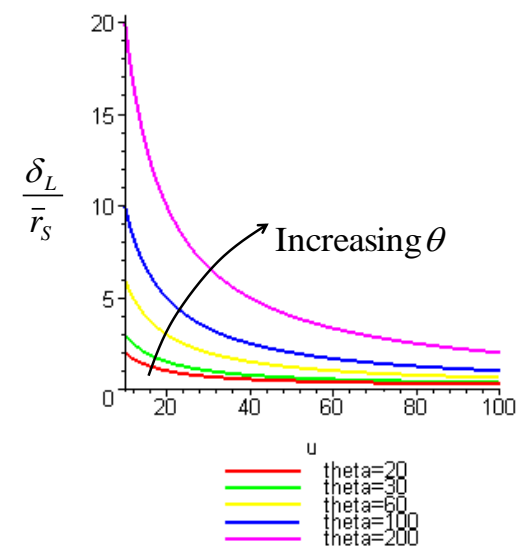

Figure 5. $\delta_{L} / \bar{r}_{S}$ as a function of $u$ for different values of $\theta$.

Moreover, based on the foregoing, one obtains $\beta_{L}=\left|\frac{d T_{l}}{d r}\right|=\frac{\bar{T}_{S}\left|\hat{p}_{C}\right|}{\bar{b} \bar{r}_{S}}(A+B)\left|\frac{E(u, \theta)}{B-\theta E(u, \theta)}\right|$

A suitable expression of the Hickman number is then

$$
H i=\left(\frac{\delta \bar{p}_{C}}{\tau r \bar{T}_{S}}\right)^{2}\left(\frac{l}{r \bar{T}_{S}}-1\right)\left[\frac{1}{\bar{T}_{S}}\left(\frac{l}{r \bar{T}_{S}}-1\right)^{2}-\frac{b\left(\bar{T}_{S}+c\right)}{\left(\bar{T}_{S}-c\right)^{3}}\right] \frac{\beta_{L} \delta_{L}^{2} \mu}{\rho_{L} \kappa_{L} \sigma^{*}}\left(\frac{1}{\bar{\rho}_{S}}-\frac{1}{\rho_{L}}\right)
$$

where $\bar{\rho}_{S}$ represents the density of the vapor at the surface of the liquid.

\section{Calculation results and conclusion}

Four pairs of propellants were studied, corresponding to eight configurations for the species of both droplet and gas: LOX- $\mathrm{H}_{2}, \mathrm{LH}_{2}-\mathrm{O}_{2}, \mathrm{LOX}-\mathrm{CH}_{4}, \mathrm{LCH}_{4}-\mathrm{O}_{2}, \mathrm{LOX}-\mathrm{C}_{10} \mathrm{H}_{22}, \mathrm{LC}_{10} \mathrm{H}_{22}-\mathrm{O} 2, \mathrm{LN}_{2} \mathrm{O}_{4}-\mathrm{MMH}$ and $\mathrm{LMMH}-\mathrm{N}_{2} \mathrm{O}_{4}$. The first letter " $\mathrm{L}$ " means "liquid" and serves to designate the species of the droplet, "MMH" means monomethyl hydrazine, and the n-decane $\mathrm{C}_{10} \mathrm{H}_{22}$ represents here the kerosene (because the dynamic model of evaporation treats only single-component drops up to now) [7].

The conditions of the study (10 bar pressure, $1000 \mathrm{~K}$ temperature) are typical of the environment of a propellant droplet in a rocket engine. Moreover, we have considered diameters of plausibly existing droplets, after the vibrational breakup owing to the shear at injection, i.e. such that the Weber number $W e=\frac{\rho\left(V_{G}-V_{L}\right)^{2}}{\sigma}$ be less than 20 (for an initial velocity difference between the gas and the droplet of $25 \mathrm{~m} / \mathrm{s}$ ). These diameters range from 1.5 micron for hydrogen to 185 microns for MMH (see figure 6). However, the conditions of evaporation equilibrium are checked for a droplet at rest. 


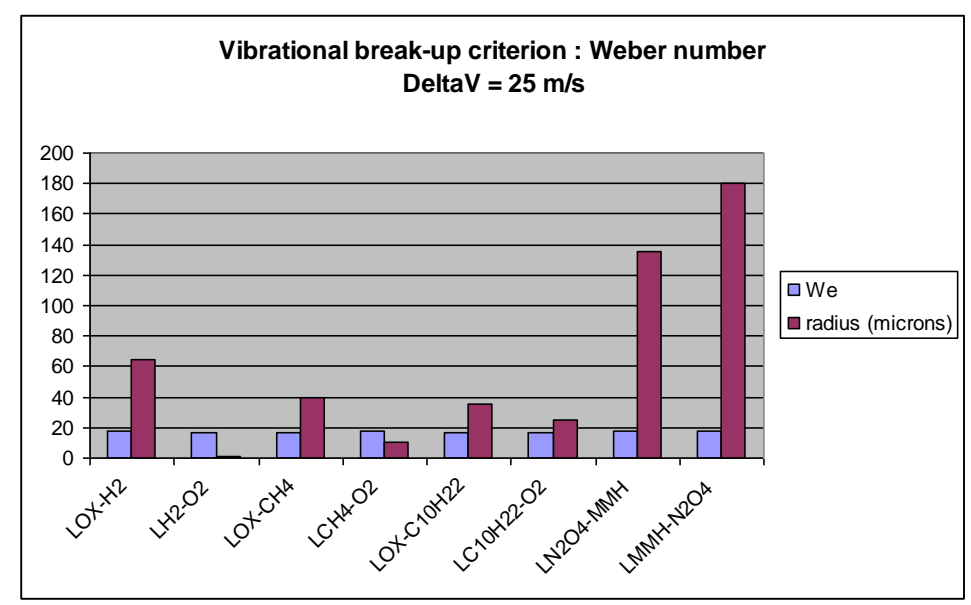

Figure 6. Droplet radii as a consequence of the vibrational break-up criterion applied in typical rocket engine conditions.

\subsection{Assumption of liquid / vapor equilibrium at the drop / gas interface.}

We have identified three necessary conditions for the liquid-vapor equilibrium at the gas-drop interface, from the consideration of the law of non-equilibrium evaporation, envisaged for a planar interface (see $\S 1.3$ ).

The first one is related to a Damköhler number of evaporation / condensation, defined as the ratio of the characteristic time of diffusion in the gas and the characteristic time of return to evaporation equilibrium:

$$
\begin{aligned}
& D a=\frac{\tau_{T}}{\tau_{\text {vap }}}=\alpha \frac{\delta}{\kappa} \sqrt{\frac{r T}{2 \pi}} \\
& \text { with } \tau_{T}=\frac{\delta^{2}}{\kappa} \text { and } \tau_{\text {vap }}=\frac{\delta \sqrt{2 \pi}}{\alpha_{\text {flow }} \sqrt{r T}} \text {. The condition is } D a=\frac{\tau_{T}}{\tau_{\text {vap }}}>>1 .
\end{aligned}
$$

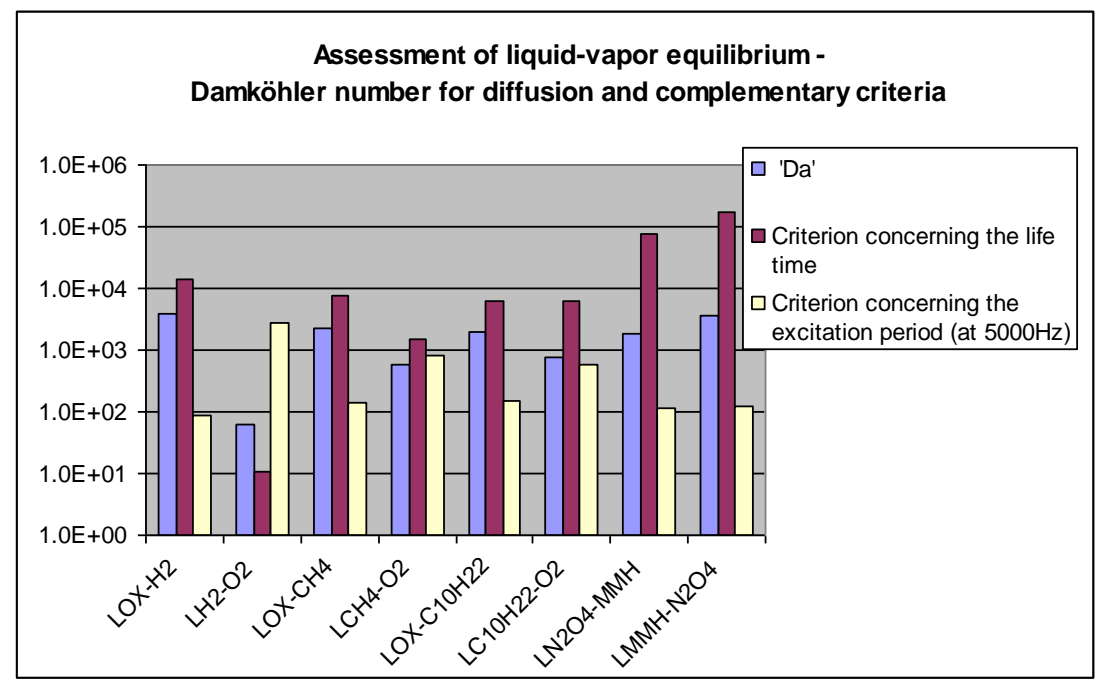

Figure 7. Checking the vapor-liquid equilibrium for a drop at rest.

Two additional conditions appear to be verified in the case of a drop subjected to an acoustic environment, regarding on the one hand the period of excitation, on the other hand the droplet lifetime:

$$
\tau_{\text {life }} / \tau_{\text {vap }}>>1, \tau_{\text {excit }} / \tau_{\text {vap }}>>1
$$


The estimates that we have carried out show that under usual conditions, at the considered frequency of 5000 $\mathrm{Hz}$ (which is a typical mediane value of the frequeny range for the combustion instabilities in liquid propellant rocket engines), these criteria are generaly properly satisfied with a difference of at least one order of magnitude on the three criteria, the lifetime criterion being however hardly satisfied in the case of an hydrogen droplet (see Figure 7).

This conclusion has nevertheless to be moderated, for three reasons.

1. We considered here the case of a drop at rest. It will be necessary to extend the analysis to the case of a drop subjected to a flow.

2. Moreover, due to the lack of data, we have assumed a unit value of the coefficients of evaporation and condensation for all species considered. These coefficients are actually neighbouring the unity for many studied species, and the others are greater than 0.01, according to the compilation done by Pound [8], which includes however none of the species of interest for us. A further literature search on the values of the coefficients of evaporation and condensation of usual propellants would thus to be carried out.

3. Finally, the triple equilibrium condition was formulated for a plane interface; it should be transposed to the case of spherical geometry.

\subsection{Hickman instability}

Hickman instability is related to differential vapor recoil, which is an inertial effect due to the difference between the mass densities of vapor and ejected liquid. Instability can occur by deformation of the interface, in case of strong evaporation non-equilibrium. One might fear that such instability occur in the case of intense noise.

Assuming a planar interface, we established the criterion of non-occurence of this instability, as a condition on the Hickman number. We determined its expression for our practical purpose (see equation (10)).

The condition of non-occurrence of instability, in the present case of a harmonic pertubation applied to a system at evaporation equilibrium, is $\mathrm{Hi} \ll<1$, whereas Palmer (see annex A.2) looked for the stability condition, considering departure from a disequilibrium state, and obtained so a critical Hickmann number depending from various dimensionless numbers.

To evaluate the expression, we estimated the parameter $b_{L}$, which represents the temperature gradient in the thermal diffusion layer of the liquid, and the characteristic time of thermal fluctuation $\tau$, using a conservative value of $10 \%$ for the relative level of pressure fluctuation $\left(\left|\hat{p}_{C}\right|=0.1\right)$ in the chamber.

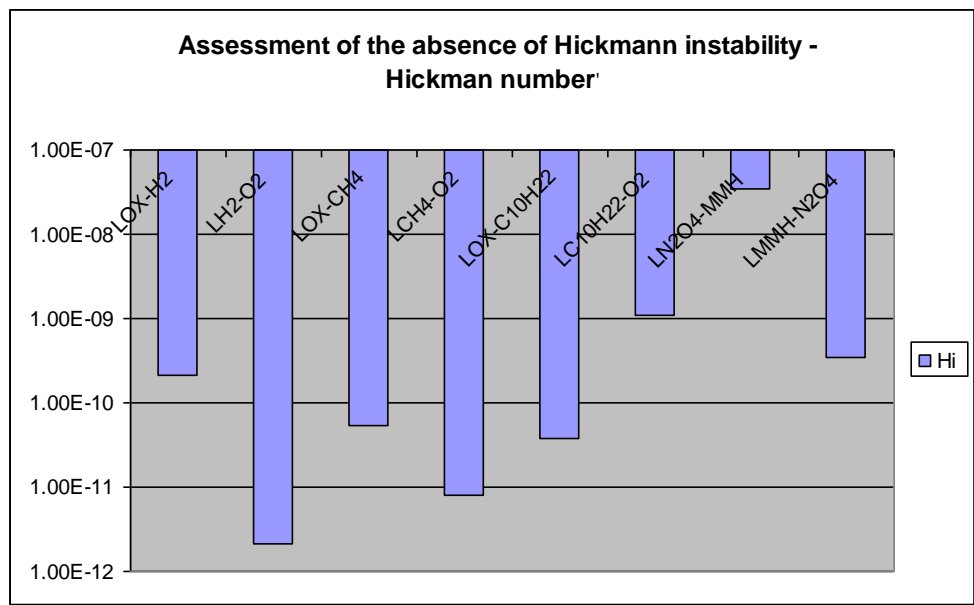

Figure 8. Values of the Hickman number for a propellant drop at rest in an environment of combustion products.

We obtained very low orders of magnitude, less than $10^{-7}$, for the Hickman number, which may suggest the absence of instability (see Figure 8). However, the results of Palmer presented in Annex A2 (figure 9) show that the problem is complex and depends on the values of several dimensionless numbers to be determined. On the 
other hand, it would be useful to translate our expression of the Hickman number in the case of the spherical interface geometry, and to take into account an external flow.

\subsection{Conclusion}

We have established criteria permitting to assess the hypothesis of local evaporation equilibrium and the absence of Hickmann instability, for an evaporating droplet submitted to acoustic excitation.

Having applied these criteria to several propellant pairs in conditions which are typical of a rocket engine, and having obtained positive results, justifies a posteriori these hypotheses, that were used to build the linear analytical model presented by the authors in reference [1] and in annex A.3.

However, in the frame of the present study the droplet was supposed at rest and we have taken only in a partial way the spherical character of the droplet geometry. The formulation of these criteria should so be extended to a droplet in a flow, and considering a fully spherical geometry. Moreover, a further literature search on the values of the coefficients of evaporation and condensation of usual propellants should be carried out.

The model presented in annex A.3 aims at evidencing the dynamic characteristics of an evaporating droplet placed at a pressure anti-node. Such a model helps to physical understanding and is a useful mean of validation for the implementation of more complex models into a fluid mechanics computer code. Moreover, the direct use of this model to study the role of evaporation in combustion instabilities, by means of a simplified approach, is in progress and will be the subject of a future publication.

\section{Appendices}

\section{A.1 The vapor recoil}

\section{A.1.1 General Equations}

The vapor recoil, which will be presented here for a species evaporating in his own vapour, results from the presence of terms to the square of velocity in the equation of momentum at the interface. When they are not negligible, they generate a pressure jump between both sides of the evaporation interface.

The equations of momentum balance at the interface without mass and without internal viscosity, projected respectively on the normal and on the tangent plane, are (see [6]):

$$
\left\{\begin{array}{l}
\dot{m}\left(\overrightarrow{\mathbf{v}}_{+}-\overrightarrow{\mathbf{v}}_{-}\right) \cdot \overrightarrow{\mathbf{N}}+p_{+}-p_{-}+\left(\overrightarrow{\overrightarrow{\boldsymbol{\Pi}}}_{+} \cdot \overrightarrow{\mathbf{N}}-\overrightarrow{\overrightarrow{\boldsymbol{\Pi}}}_{-} \cdot \overrightarrow{\mathbf{N}}\right) \cdot \overrightarrow{\mathbf{N}}=-\sigma \vec{\nabla} \cdot \overrightarrow{\mathbf{N}} \\
\dot{m}\left(\overrightarrow{\mathbf{v}}_{+/ /}-\overrightarrow{\mathbf{v}}_{-/ /}\right)+\left(\overrightarrow{\overrightarrow{\boldsymbol{\Pi}}}_{+} \cdot \overrightarrow{\mathbf{N}}-\overrightarrow{\overrightarrow{\boldsymbol{\Pi}}} \cdot \overrightarrow{\mathbf{N}}\right)_{/ /}=\vec{\nabla}_{S} \sigma
\end{array} \quad \text { with } \vec{\nabla}_{S}=\vec{\nabla}_{/ /}\right.
$$

In these equations, the liquid side is designated by the index (-) and the vapor side by the index $(+), \overrightarrow{\mathbf{N}}$ is the unit normal directed from - to,$+ \dot{m}$ is the mass flow rate through the interface unit area, $\overrightarrow{\mathbf{v}}$ is the velocity vector, $p$ is the thermodynamic pressure, $\overrightarrow{\overrightarrow{\boldsymbol{\Pi}}}$ is the tensor of viscous pressure and $\sigma$ the surface tension.

The equation of mass balance is written

$$
\dot{m}=\rho_{-}\left(\overrightarrow{\mathbf{v}}_{-}-\overrightarrow{\mathbf{w}}\right) \cdot \overrightarrow{\mathbf{N}}=\rho_{+}\left(\overrightarrow{\mathbf{v}}_{+}-\overrightarrow{\mathbf{w}}\right) \cdot \overrightarrow{\mathbf{N}},
$$

where $\rho$ is the density and $\overrightarrow{\mathbf{w}}$ the interfacial velocity.

\section{A.1.2 Planar interface}

For a planar interface, the curvature $\vec{\nabla} \cdot \overrightarrow{\mathbf{N}}$ is equal to zero. So for inviscid fluids, equations (13) and (14) result in: 
$\left\{\begin{array}{l}\dot{m}\left(\overrightarrow{\mathbf{v}}_{+}-\overrightarrow{\mathbf{v}}_{-}\right) \cdot \overrightarrow{\mathbf{N}}+p_{+}-p_{-}=0 \\ \dot{m}=\rho_{-}\left(\overrightarrow{\mathbf{v}}_{-}-\overrightarrow{\mathbf{w}}\right) \cdot \overrightarrow{\mathbf{N}}=\rho_{+}\left(\overrightarrow{\mathbf{v}}_{+}-\overrightarrow{\mathbf{w}}\right) \cdot \overrightarrow{\mathbf{N}}\end{array}\right.$

We deduce the pressure jump at the interface

$$
p_{-}-p_{+}=\dot{m}^{2}\left(\frac{1}{\rho_{+}}-\frac{1}{\rho_{-}}\right) \text {. }
$$

As below the critical point we generally have $\rho_{+}<<\rho_{-}$, equation (16) becomes

$$
p_{-}-p_{+}=\rho_{+}\left(v_{+\perp}-w_{\perp}\right)^{2},
$$

showing that the recoil force is exerted to the liquid.

Let us now consider the constitutive law of evaporation.

- At evaporation-condensation equilibrium:

- In the absence of recoil, there is equality of thermodynamic potentials of liquid and vapor, which one translates for a pure substance as $g_{+}=g_{-}$,

where we have $g_{-}=g_{T_{-}}^{0}+\left(p_{-}-p_{0}\right) / \rho_{-}, \quad g_{+}=g_{T_{+}}^{0}+R T \ln p_{+} / p_{0} \quad$ with $\quad p_{0}$ standard pressure, $T_{+}=T_{-}=T, \quad \rho_{-}=\rho_{-}(T)$ which is assumed to be constant;

- With recoil force, we get $g_{+}=g_{-}-\left(v_{+\perp}-w_{\perp}\right)^{2} / 2+\left(v_{-\perp}-w_{\perp}\right)^{2} / 2$.

- $\quad$ Near evaporation-condensation equilibrium (assuming $T_{+}=T_{-}=T_{S}=T$ ):

- In the absence of recoil, we obtain the constitutive law $\dot{m}=-\frac{L}{T}\left(g_{+}-g_{-}\right)$,

where $L$ is a phenomenological coefficient;

- With recoil force, we should have: $\dot{m}=-\frac{L}{T}\left(g_{+}-g_{-}+\left(v_{+\perp}-w_{\perp}\right)^{2} / 2-\left(v_{-\perp}-w_{\perp}\right)^{2} / 2\right)$.

- Far from evaporation-condensation equilibrium, the unit rate is given by a relation of type (2) or (3).

\section{A.1.3 Curvature effect}

For a curved interface, the effect of surface tension must be added:

$$
p_{-}-p_{+}=\dot{m}\left(v_{+\perp}-v_{-\perp}\right)+\sigma \nabla \cdot \mathbf{N}
$$

Assuming near-equilibrium, the evaporation rate writes

$$
\dot{m}=-\frac{L}{T}\left[g_{+}-g_{-}+\frac{1}{2}\left(v_{+\perp}-w_{\perp}\right)^{2}-\frac{1}{2}\left(v_{-\perp}-w_{\perp}\right)^{2}\right]
$$

\section{A.2 The Hickman instability}


Palmer studied the instability resulting from the recoil of the vapor in the case of a pure liquid under reduced pressure by taking, as stable reference state, a horizontal flat evaporation surface [4]. This instability is called Hickman instability [5]. Palmer [4] shows that "rapidly evaporating liquid is unstable for local variations of the evaporation rate, local depressions of the surface being produced by the force exerted on the surface by vapor evaporation and rapid flow of liquid being caused by the resulting shear exerted on the liquid surface by the vapor."

Densities of both liquid and vapor are assumed to be constant and uniform. It is also supposed that, in the absence of instabilities due to surface tension, a stationary thermal boundary layer thickness $\delta_{L}$, through which heat is transported by conduction only, exists in the liquid, in the vicinity of the interface. The temperature profile in the thermal boundary layer is assumed to be linear and the temperature of the liquid outside of the boundary layer is assumed to be constant. In addition, the cooling rate of the liquid surface by heat conduction in the vapor phase is assumed to be negligible in comparison to the heat removed by the phase change (Figure 3 ).

We write first the balance equations for the reference state, denoted by $(*)$, in which all quantities are constant. The mass flow rate is given by the relation (3), which is written in the following way:

$$
\dot{m}^{*}=\alpha\left(\frac{\mathcal{M}}{2 \pi R}\right)^{1 / 2}\left(\frac{p^{0}}{\left(T_{S}\right)^{1 / 2}}-\frac{p_{G}{ }^{*}}{\left(T_{G}{ }^{*}\right)^{1 / 2}}\right)
$$

where $\alpha$ is a coefficient of evaporation, $\mathcal{M}$ the molar weight of the liquid, $R$ the universal gas constant, $p^{0}$ the equilibrium vapor pressure at the surface temperature $T_{S}$, which is equal to the temperature $T_{L}{ }^{*}$ of the liquid, $p_{G}{ }^{*}$ and $T_{G}{ }^{*}$ the pressure and the temperature of the gaseous phase above the liquid. The interface is devoid of mass and viscosity, but has a surface tension.

Balance equations of mass, momentum and energy write respectively:

$$
\left\{\begin{array}{l}
\dot{m}^{*}=\rho_{L} W_{L}{ }^{*}=\rho_{G} W_{G}{ }^{*} \\
p_{L}{ }^{*}-p_{G}{ }^{*}=\left(\dot{m}^{*}\right)^{2}\left(\frac{1}{\rho_{G}}-\frac{1}{\rho_{L}}\right) \\
\dot{m}^{*} l+\frac{1}{2}\left(\dot{m}^{*}\right)^{3}\left(\frac{1}{\rho_{G}{ }^{2}}-\frac{1}{\rho_{L}{ }^{2}}\right)+\lambda_{L} \frac{\partial T_{L}}{\partial z}=0
\end{array}\right.
$$

where $l$ is the latent heat of vaporization at temperature $T_{L}{ }^{*}$ and $W$ the vertical component of the velocity vector.

For the perturbed state, we define the perturbation $f^{\prime}$ of $f$ by setting $f=f^{*}+f^{\prime}$, and we write the system of equations of the linearized problem for small perturbations.

Writing the equation of the disturbed surface

$$
z=\eta(x, y, t)
$$

we obtain successively the unit normal pointing from liquid to gas, the average normal curvature and the normal velocity of the interface:

$$
\overrightarrow{\mathbf{N}}=\overrightarrow{\mathbf{k}}-\vec{\nabla} \eta, \quad \vec{\nabla} \cdot \overrightarrow{\mathbf{N}} \cong-\vec{\nabla}_{/ /}^{2} \eta, \quad w \cong \partial \eta / \partial t
$$

where $\overrightarrow{\mathbf{k}}$ is the unit vertical upward vector.

Equations for small perturbations of mass, momentum and energy write as follows: 


$$
\left\{\begin{array}{l}
\dot{m}^{*}\left(\frac{1}{\rho_{G}}-\frac{1}{\rho_{L}}\right) \vec{\nabla}_{/ / \eta}^{2}=\frac{\partial W_{L}^{\prime}}{\partial z}-\frac{\partial W_{G}^{\prime}}{\partial z} \\
p_{L}{ }^{\prime}-p_{G}{ }^{\prime}+2 \dot{m}^{*} \dot{m}^{\prime}\left(\frac{1}{\rho_{G}}-\frac{1}{\rho_{L}}\right)+2\left(\mu_{L} \frac{\partial W_{L}^{\prime}}{\partial z}-\mu_{G} \frac{\partial W_{G}^{\prime}}{\partial z}\right)+g\left(\rho_{L}-\rho_{G}\right) \eta-\sigma^{*} \vec{\nabla}_{/ /}^{2} \eta=0 \\
\vec{\nabla}_{/ /}^{2} \sigma^{\prime}=\mu_{L}\left(\vec{\nabla}_{/ /}^{2} W_{L}^{\prime}{ }_{L}-\frac{\partial^{2} W_{L}^{\prime}}{\partial z^{2}}\right)-\mu_{G}\left(\vec{\nabla}_{/ /}^{2} W_{G}^{\prime}-\frac{\partial^{2} W_{G}^{\prime}}{\partial z^{2}}\right) \\
\dot{m}^{\prime} l+\lambda_{L} \frac{\partial T_{L}^{\prime}}{\partial z}+\frac{3}{2}\left(\dot{m}^{*}\right)^{2} \dot{m}^{\prime}\left(\frac{1}{\rho_{G}^{2}}-\frac{1}{\rho_{L}^{2}}\right)-2 \dot{m}^{*}\left(\frac{\mu_{G}}{\rho_{G}} \frac{\partial W_{G}^{\prime}}{\partial z}-\frac{\mu_{L}}{\rho_{L}} \frac{\partial W_{L}^{\prime}}{\partial z}\right)=0
\end{array}\right.
$$

In the first momentum equation, the first term is the pressure jump, whereas the second one corresponds to the recoil force, the third one to the viscous forces, the fourth one to gravity; the last one is the surface tension.

For a local depression we have $\eta<0$, and $\vec{\nabla}_{/ /}^{2} \eta>0$, then the fourth and fifth terms are both negative. Hence when the interface is hollow, the sum of the first three terms is positive. These three combined forces are responsible for the instability, leading to an increase of the surface depression.

Far from the interface all perturbations are assumed by Palmer to be nearly nil:

$$
\left\{\begin{array}{lll}
W_{L}^{\prime}=\partial W_{L}^{\prime} / \partial z=T_{L}^{\prime}=0 & \text { as } & z \rightarrow-\infty \\
W_{G}^{\prime}{ }_{G}=\partial W_{G}^{\prime} / \partial z=0 & \text { as } & z \rightarrow+\infty
\end{array}\right.
$$

Palmer analyzes the problem of small perturbations of the form $\varphi^{\prime}(x, y, z, t)=e^{\gamma t} f(x, y) \varphi(z)$, where $\gamma$ is the growth rate constant and $f$ satisfies the wave equation

$$
\nabla^{2} f+K^{2} f=0 .
$$

$K$ is the wave number. The neutral stability requires that the real part of the growth rate constant $\gamma$ be zero but if only the "stationary" (i.e., non-oscillatory) modes of instability are considered, as Palmer does, the imaginary part of $\gamma$ is also equal to zero. The dispersion equation relates the Hickman number $H i=\left(\frac{d \dot{m}}{d T}\right)^{*} \frac{\dot{m}^{*} \beta_{L} \delta_{L}^{2} \mu_{G}}{\rho_{L} \kappa_{L} \sigma^{*}}\left(\frac{1}{\rho_{G}}-\frac{1}{\rho_{L}}\right)$, defined by equation (7), to the dimensionless wave number $K$ and other dimensionless groups.

The latter are the Marangoni number $M a=(-d \sigma / d T)\left(\beta_{L} \delta_{L}{ }^{2} /\left(\kappa_{L} \mu_{L}\right)\right)$, the crispation number $C r=\mu_{L} \kappa_{L} /\left(\sigma^{*} \delta_{L}\right)$, the viscosity ratio $\mu_{L} / \mu_{G}$, the density ratio $\rho_{L} / \rho_{G}$, the Reynolds number $\operatorname{Re}=\dot{m}^{*} \delta_{L} / \mu_{L}$, the Prandtl number $\operatorname{Pr}=\mu_{L} /\left(\rho_{L} \kappa_{L}\right)$, the Bond number $B o=\delta_{L}{ }^{2} g\left(\rho_{L}-\rho_{G}\right) / \sigma^{*}$ and the Brinkman number $B r=\dot{m}^{*} \mu_{L}^{2} /\left(\beta_{L} \kappa_{L} \rho_{L}^{2} \delta_{L}^{2}\right)$.

In these groups, $\left(-\beta_{L}\right)$ refers to the thermal gradient in the thermal boundary layer thickness $\delta_{L}$ (Figure 3), $(d \dot{m} / d T)$ is the rate of variation of the evaporation rate as a function of the interfacial temperature and $\kappa_{L}$ is the thermal diffusivity.

The figure 9, redrawn from Palmer's paper, shows respective influences of some of these dimensionless numbers on the neutral stability curve, in case vapor recoil prevails. 

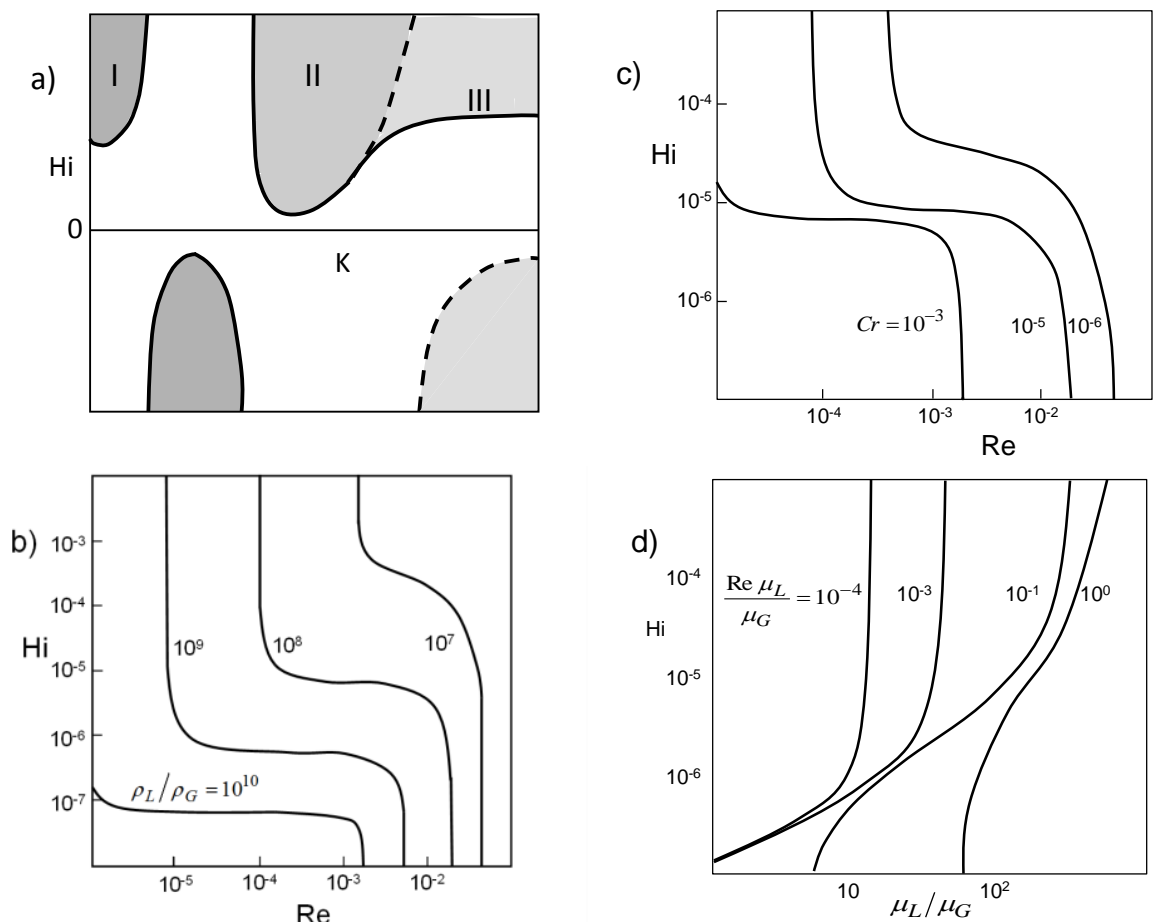

Figure 9: Critical Hickman number. a) Typical results. Zone I: mechanism of moving boundary, zone II: vapor recoil, zone III: viscous dissipation, dotted curve: neutral stability curve for $\mathrm{Br}=0 . \mathrm{b}$ ) Dependency on the Reynolds number for different values of the density ratio, $\mathrm{Cr}=10^{-5}, \mathrm{Bo}=1, \mathrm{Pr}=10, \mu_{L} / \mu_{G}=10^{2}, \mathrm{Br}=0, \mathrm{Ma}=0$. c) Same for different $\mathrm{Cr}, \mathrm{Bo}=1$,

$\left.\operatorname{Pr}=10, \mu_{L} / \mu_{G}=10^{2}, B r=0, M a=0 . d\right)$ Dependence on the ratio of viscosities for various values of the Reynolds number of the vapor, $C r=10^{-5}, B o=1, \operatorname{Pr}=10, \rho_{L} / \rho_{G}=10^{8}, B r=0, M a=0$ (redrawn from Palmer [4]).

\section{A.3 Linear approach of the evaporation dynamics of a drop submitted to acoustic excitation}

We consider a droplet supplied with the flow $\overline{\dot{M}}$ equal to the mean evaporation flow rate $\overline{\dot{M}}$ (Figure 10), considered as beeing representative of a mean droplet at a defined point of a combustion chamber ${ }^{7}$.

In Heidmann's theory [9], the temperature of the drop is assumed to be uniform (assuming infinite conductivity) and equal to the temperature $T_{S}$ of its surface, which is in local evaporation equilibrium with the external gas mixture. More recently, a continuous temperature field was considered (with a finite thermal diffusivity $\kappa_{L}$ ) [1].

The droplet is disturbed by high frequency waves coming from the combustion chamber; we characterize its response by a response factor, neglecting the feed-back on incident disturbances.

We consider here what happens at an acoustic velocity node of the combustion chamber, which is also a pressure antinode. This means that the incident disturbance concerns the pressure $p_{c}$. The spherical drop evaporates so in a medium that is at rest at infinity, and which imposed conditions are chamber ones.

\section{A.3.1 Equations for the gaseous phase}

The unperturbed state is a stable situation for which any thermodynamic variable $f$ of droplet has a uniform distribution $\bar{f}$.

For small harmonic disturbances, we set $f=\bar{f}+\Delta f, f^{\prime}=\Delta f / \bar{f}$ and $f^{\prime}=\hat{f}(x) e^{i \omega t}$. We show that the evolution is governed by two equations:

\footnotetext{
${ }^{7}$ The model of supplied drop is due to Heidmann. It allows to represent a two-phase flow by a mean drop which feeding corresponds to providing fresh drops at the same place of the considered combustion chamber. The mean diameter of this average drop is considered to be invariant, as the liquid supply compensates the evaporation.
} 


$$
\begin{aligned}
& \hat{\dot{M}}=\bar{\alpha} \frac{i u}{1+i u}\left(\bar{b} \hat{T}_{S}-\hat{p}_{C}\right) \\
& \Delta \hat{Q}_{L}=\overline{\dot{M}} \bar{\ell}\left(\bar{a} \hat{p}_{C}-\mu \hat{T}_{S}\right)
\end{aligned}
$$

In these equations, $\dot{M}, Q_{L}, T_{s}, p_{c}, \ell$ are respectively the mass flow rate of evaporation, the heat flow penetrating into the drop from the surrounding gas mixture ${ }^{8}$, the surface temperature, the pressure chamber and the latent heat of evaporation. The coefficients used in these equations are:

$$
\left\{\begin{array}{l}
\bar{\alpha}=\frac{\bar{B}_{M}}{\left(1+\bar{B}_{M}\right) \ln \left(1+\bar{B}_{M}\right)} \frac{\bar{Y}_{A C} \bar{Y}_{F S}}{\bar{Y}_{A S}\left(\bar{Y}_{F S}-\bar{Y}_{F C}\right)} \frac{\mathcal{M}_{F}}{\mathcal{M}_{F} \bar{X}_{F S}+\mathcal{M}_{A} \bar{X}_{A S}}, \bar{a}=\frac{\bar{T}_{C}}{\bar{T}_{C}-\bar{T}_{S}} \frac{\gamma-1}{\gamma}+\varphi \\
\bar{b}=\frac{\bar{T}_{S}}{\left(\bar{T}_{S}-c\right)^{2}} b, \mu=\frac{\bar{T}_{S}}{\bar{T}_{C}-\bar{T}_{S}}-\frac{2 c}{\bar{T}_{S}-c}+\bar{b} \varphi
\end{array}\right.
$$

In these definitions, $F$ designates the fuel, $A$ represents the burnt gases, $C$ the chamber and $S$ the surface of the drop, the quantities $Y_{j}, B_{M}, \mathcal{M}_{j}, \gamma$ are respectively the mass fraction of species $j$, the Spalding parameter for the mass, the molecular weight of species $j$ and the isentropic coefficient (assumed to be constant ${ }^{9}$ ). The coefficients $b$ and $c$ are derived from the expression of the latent heat given in the form: $\ell=b R T_{S}^{2} / M_{F}\left(T_{S}-c\right)^{2} \cdot \varphi$ corresponds to the function $\varphi=\bar{Y}_{A C} \bar{Y}_{F S} \mathcal{M}_{F} / \bar{Y}_{A S}\left(\bar{Y}_{F S}-\bar{Y}_{F C}\right)\left(\mathcal{M}_{F} \bar{X}_{F S}+\mathcal{M}_{A} \bar{X}_{A S}\right)$.

\section{A.3.2. Equations of the liquid phase}

The following equation for small perturbations concerns the temperature of the liquid.

It is written according to the classical irreversible thermodynamics, neglecting the thermal convection due to injection, provided that the characteristic time of internal thermal conduction is small in comparison to the residence time of the fluid in the droplet [1]:

$$
\frac{\partial T_{l}^{\prime}}{\partial t}-\frac{\kappa_{L}}{r} \frac{\partial^{2}\left(r T_{l}^{\prime}\right)}{\partial r^{2}}=0
$$

Setting $T^{\prime}{ }_{l}=\hat{T}_{l}(r) e^{i \omega t}$, we find the relation $i \omega r \hat{T}_{l}-\kappa_{L} \partial^{2}\left(r \hat{T}_{l}\right) / \partial r^{2}=0$, which characteristic equation writes $\left(s \bar{r}_{S}\right)^{2}-i \frac{3 u}{\theta}=0$.

We define here the reduced frequency $u=3 \omega \bar{\tau}_{v}$, the residence time in the fed drop $\bar{\tau}_{\mathrm{v}}=\bar{M} / \overline{\dot{M}}$ (which is proportional to the lifetime of the corresponding free drop), the time of heat conduction $\tilde{\tau}_{T}=\bar{r}_{S}{ }^{2} / \kappa_{L}$, and $\theta=\bar{\tau}_{v} / \tilde{\tau}_{T}$. The eigenvalues $s^{+} \bar{r}_{S}, s^{-} \bar{r}_{S}$ are such that $s^{+} \bar{r}_{S}=s_{0} \bar{r}_{S}, s^{-} \bar{r}_{S}=-s_{0} \bar{r}_{S}$, with $s_{0} \bar{r}_{S}=(1+i) \sqrt{3 u / 2 \theta}$ and $r T_{l}=C^{+} e^{s_{0} r}+C^{-} e^{-s_{0} r}$.

The boundary conditions are respectively:

- $T_{l}{ }^{\prime}=T_{s}{ }^{\prime}$ and $\Delta Q_{L}=4 \pi \bar{r}_{S}{ }^{2} k_{L} \bar{T}_{S} d T_{l}{ }^{\prime} /\left.d r\right|_{\bar{r}_{s}}$ at the surface of drop,

- $d T_{l} /\left.d r\right|_{r=0}=0$ at the center of drop, assuming adiabatic feeding.

For small harmonic disturbances, we finally find:

\footnotetext{
${ }^{8}$ We have: $Q_{L}=Q-\dot{M} \ell$ where $Q$ is the heat flux form the gas to the drop, and $\bar{Q}_{L}=0$ in the reference unperturbed state; the absolute perturbation of $Q_{L}$ is thus $\Delta Q_{L}=Q_{L}$, but one cannot write $Q_{L}{ }^{\prime}=\Delta Q_{L} / \bar{Q}_{L}$.

${ }^{9}$ Small perturbations are assumed to propagate in the gaseous phase in an isentropic way, at the sound velocity.
} 


$$
\begin{aligned}
& r \hat{T}_{l}=\bar{r}_{S} \hat{T}_{S} \frac{e^{s_{0} r}-e^{-s_{0} r}}{e^{s_{0} \bar{T}_{S}}-e^{-s_{0} \bar{T}_{S}}} \\
& \Delta \hat{Q}_{L}=4 \pi \bar{r}_{S} k_{L} \bar{T}_{S} \hat{T}_{S} E(u, \theta)
\end{aligned}
$$

with $E(u, \theta)=1-s_{0} \bar{r}_{S} \operatorname{coth}\left(s_{0} \bar{r}_{S}\right)$.

\section{A.3.3 Transfer function and response factor}

We define the complex transfer function by $Z=\hat{\dot{M}} / \bar{\alpha} \hat{p}_{C}$.

The expression of this function,

$$
Z=\frac{i u}{1+i u} \frac{A+\theta E(u, \theta)}{B-\theta E(u, \theta)},
$$

is obtained by eliminating $\Delta \hat{Q}$ and $\hat{T}_{S}$ between equations (31), (32) and (35) and setting $\lambda=c_{L} \bar{T}_{S} / \bar{\ell}$, $A=3(\bar{a} \bar{b}-\mu) / \lambda$, and $B=3 \mu / \lambda$.

The response factor is defined by $N=\iint_{V, t} q^{\prime}(V, t) p^{\prime}(V, t) d t d V / \iint_{V, t}\left(p^{\prime}(V, t)\right)^{2} d t d V$, where $p^{\prime}$ is the relative intensity of the incident disturbance (here that of the pressure chamber) and $q^{\prime}$ the relative intensity of the response (here the rate of evaporation). We have: $p^{\prime}=p_{c}{ }^{\prime}=\left(p_{c}-\bar{p}_{c}\right) / \bar{p}_{c}, q^{\prime}=(\dot{M}-\overline{\dot{M}}) / \overline{\dot{M}}$. We show that for harmonic disturbances,

$$
N=\bar{\alpha} \mathfrak{R}(Z) .
$$

An amplification effect or a damping effect occurs, respectively when the response factor is positive or negative. The cutoff reduced pulsation $u_{c}$ separates the two regimes (Figure 10).

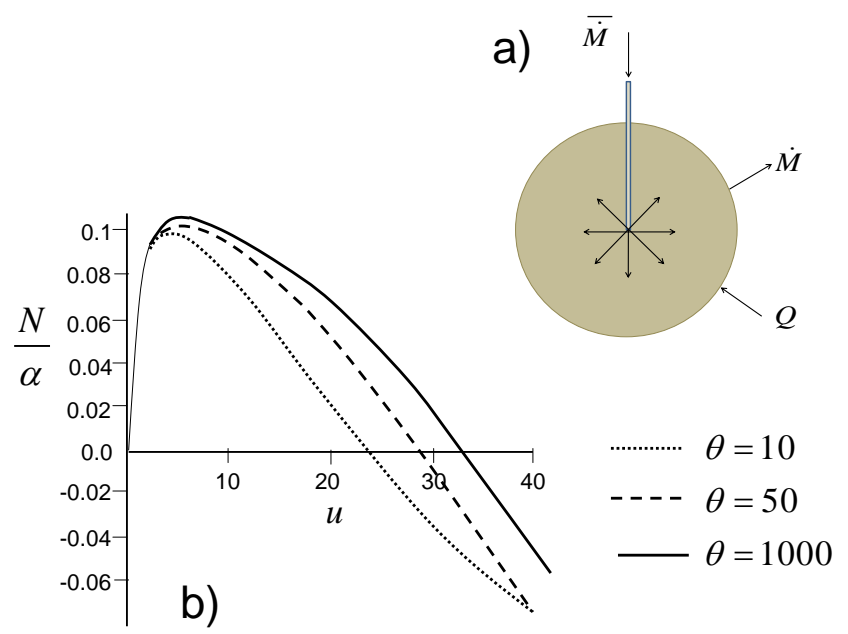

Figure 10: a) The vaporizing droplet of Heidmann, continuously supplied by a steady flow rate. b) The reduced response factor as a function of the reduced frequency (for arbitrary values $A=10, B=100$ ).

\section{Acknowledgements}


This study was co-funded by CNES (French Space Agency) and ONERA and was performed in the framework of CNES-ONERA French Research \&Technology activities on the high frequency combustion stability of liquid propellant rocket engines ; it is a contribution to the French-Germany program implying six partners: Astrium, CNES, CNRS, DLR, ONERA and SNECMA.

\section{Reference list}

[1] Prud'homme, R., Habiballah, M., Matuszewski, L., Mauriot, Y., Nicole, A.: Theoretical analysis of transient heating and dynamic response of a vaporizing droplet to acoustic oscillations. Journal of Propulsion and Power vol. 26 no. 1, 74-83, 2010

[2] Prud'homme, R. (2010): Flows of Reactive Fluids, FMIA Series 94, Springer.

[3] Bond, M., Struchtrup, H.: Mean evaporation and condensation coefficients based on energy dependent condensation probability, Physical Review E 70, 061605 (2004).

[4] Palmer, H.J.: The hydrodynamic stability of rapidly evaporating liquids at reduced pressure, J. Fluid Mech., 75, 487-511 (1976).

[5] Hickman, K.: Surface behaviour in the pot still, Industrial and Enginering Chemistry, 44(2) :1892$1902(1952)$.

[6] Gatignol R. \& Prud'homme R. : Mechanical and thermodynamical modeling of fluid interfaces, Series on Advances in Mathematics for Applied Sciences - Vol. 58, World Scientific (2001)

[7] Mauriot Y., Prud'homme R., ONERA Report RT 5/16530 DEFA-Décembre 2011 (Onera-Cnes Confidential).

[8] Pound, G.M.: Selected values of evaporation and condensation coefficients for simple substances. Phys. Chem. Ref. Data, Vol. 1, No. 1, 1972

[9] Heidmann, M. F. \& Wieber P. R.: Analysis of frequency response characteristics of propellant vaporization, NASA Technical Note D-3749 (1966). 